\title{
Vasomotor Symptoms: More Than Temporary Menopausal Symptoms
}

\author{
Ki-Jin Ryu ${ }^{1}$, Hyuntae Park ${ }^{1}$, Jin Seol Park², Yeon Woo Lee ${ }^{2}$, Soo Young Kim², Hayun Kim², Youngmi Jeong ${ }^{1}$, Yong Jin Kim \\ Kyong Wook Yi ${ }^{1}$, Jung Ho Shin ${ }^{1}$, Jun Young Hur ${ }^{1}$, Tak Kim \\ ${ }^{1}$ Department of Obstetrics and Gynecology, Korea University College of Medicine, Seoul, Korea, ${ }^{2}$ Korea University College of Medicine, \\ Seoul, Korea
}

Vasomotor symptoms (VMS), such as hot flashes and night sweating, are classic menopausal symptoms experienced by a majority of perimenopausal and postmenopausal women. VMS have received a great deal of attention due to their relationship with cardiometabolic risk. Further, accumulating evidence indicates that VMS are associated with an increased risk of several chronic diseases, including metabolic syndrome, type 2 diabetes mellitus, nonalcoholic fatty liver diseases, and osteoporosis in perimenopausal and postmenopausal women. These findings suggest VMS as biomarkers of impaired cardiometabolic conditions rather than just temporary symptoms in menopausal women, warranting further studies to confirm the casual relationship of VMS with these diseases and the exact underlying mechanism in this context.

Key Words: Cardiovascular diseases, Hot flashes, Menopause, Metabolic syndrome, Vasomotor symptoms

\section{INTRODUCTION}

Due to the increase in average life expectancy, mainly in developed countries, most women spend more than one-third of their lifetime in a postmenopausal state. The mean life expectancy at birth of Korean women was 85.7 years in 2018, 2.4 years longer than that in Organisation for Economic Co-operation and Development countries [1]. Health management and quality of life during the postmenopausal period have received more attention in recent years. Vasomotor symptoms (VMS) in particular, such as hot flashes and night sweating, are major issues experienced by menopausal women (Fig. 1); in fact, $50 \%$ to $75 \%$ of perimenopausal and postmenopausal women experience some degree of VMS during their lifetime [2]. Unlike traditionally, where VMS were regarded as transient symptoms of perimenopause, recent studies have suggested that VMS can persist for more than seven years after onset

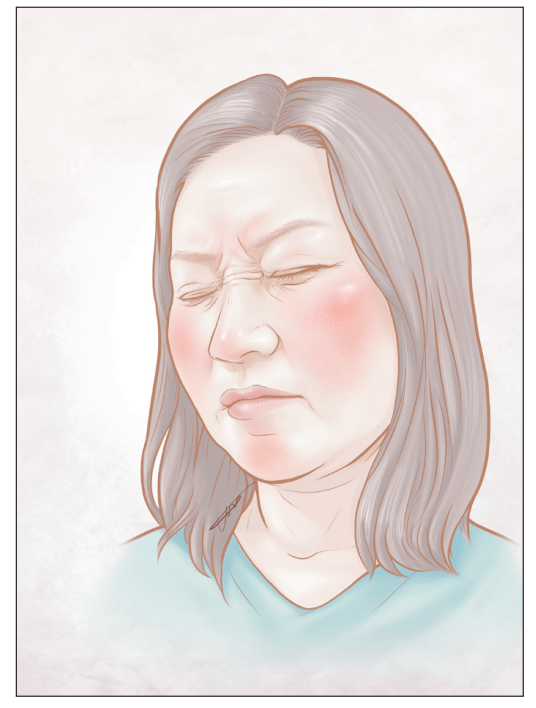

Fig. 1. Vasomotor symptoms in menopausal women. 
of menopause [3]. International guidelines recommend hormone replacement therapy (HRT) for management of VMS in selected cases $[4,5]$. Based on recent findings on the role of kisspeptin/neurokinin B/dynorphin neuron, a modulator of gonadotropin-releasing hormone secretion, in the physiology of VMS, neurokinin 3 receptor antagonists stand out as a novel treatment option for VMS, and ongoing clinical trials are aiming to confirm their efficacy [6].

Postmenopausal women are at a higher risk of developing several chronic diseases, including obesity, metabolic syndrome, cardiovascular diseases (CVDs), and osteoporosis, than premenopausal women independent of age [7]. Decreasing sex hormone levels during menopause have been collectively considered a key factor in the association between these chronic diseases and menopause; however, the exact mechanisms underlying these associations remain unclear. Recent accumulating evidence indicates that VMS are not only bothersome symptoms of menopausal transition, but also indicators of risk of the aforementioned chronic diseases and related conditions. Epidemiologic studies conducted by our study group have shown that presence or severity of VMS is associated with increased risk of metabolic syndrome, insulin resistance, nonalcoholic fatty liver disease (NAFLD), and osteoporosis [8-10]. However, the exact underlying mechanisms of those associations are unclear, and many confounding factors require consideration, such as age, obesity, and lifestyle choices [11].

This study aimed to review previous investigations of the associations between VMS and chronic diseases among perimenopausal and postmenopausal women and to identify whether VMS collectively are an independent risk factor for those diseases. Further, our review aimed to examine whether VMS collectively could be an indicator of those diseases and be addressed to prevent and reduce patient risk.

\section{VMS AND OBESITY}

Previous studies have suggested that obesity might have a protective effect against VMS because androgens are aromatized into estrogens by aromatase cytochrome P450 in adipose tissue [12]. However, recent accumulated evidence showed that women with obesity are more likely to report VMS than lean women $[13,14]$. A subcohort of a large-sized, longitudinal cohort study suggested that the association between VMS and obe- sity may change with menopausal stage (e.g., positive association at early menopausal transition, inverse association at late menopausal transition) [15]; however, these findings need to be confirmed by further epidemiologic studies.

One of the generally accepted hypotheses for these associations is that the insulating effect of increased body fat in women with obesity can raise the core body temperature and may facilitate the association between obesity and VMS [16]. Another suggested hypothesis is that chronic inflammation in adipose tissue triggered by obesity may negatively affect ovarian function and induce VMS in the menopausal transition [17]. Supporting this hypothesis, it was shown that leptin, a proinflammatory adiponectin, is associated with VMS, whereas anti-inflammatory adiponectin was not [18]. Further, abundant body fat may influence the central nervous system and increase sympathetic tone, i.e., the thermoregulatory variable contributes to an increase in hot flashes [19]. However, these explanations have not yet been verified, and the exact underlying mechanism of the association between obesity and VMS should be revealed by further investigations.

\section{VMS AND METABOLIC SYNDROME}

Metabolic syndrome constitutes a group of metabolic abnormalities including increased blood pressure, increased triglyceride level, decreased high-density lipoprotein (HDL) cholesterol level, hyperglycemia, and central obesity that increase an individual's risk of CVDs and diabetes mellitus (DM) [20]. The prevalence of metabolic syndrome is increasing both worldwide and in Korea due to changing lifestyle [21]. Menopause is a well-known risk factor of an increased prevalence of metabolic syndrome independent of age [22]. Further, several epidemiologic studies have reported that postmenopausal status is associated with increased risk of metabolic syndrome as well as VMS in menopausal women $[7,11,14]$. However, most existing studies on these relationships are limited by small population size or cross-sectional study design. Thus, the causal relationship and underlying mechanism should be elucidated in further studies.

According to the accumulated evidence, menopausal VMS are diversely associated with each component of metabolic syndrome. Our study group reported the results of a cross-sectional study of 1,906 Korean postmenopausal women suggesting that the presence 
of VMS is associated with increased risk of metabolic syndrome after adjustment for confounding factors [14]. Further, lipid abnormalities and central obesity are important metabolic components associated with these symptoms [14]. In a previous study involving data from the Korean National Health and Nutrition Examination Survey, both lipid abnormalities and central obesity were major factors related to increased prevalence of metabolic syndrome in the Korean population over the past 10 years [21]. Further studies on the association between VMS and metabolic syndrome in Korean women may require an emphasis on these two metabolic components. In the Study of Women's Health Across the Nation (SWAN), the presence and frequency of VMS were associated with higher triglyceride, low-density lipoprotein cholesterol, and HDL cholesterol levels [23]. Of note, night sweats were associated with abnormal lipid profiles in menopausal women in a previous epidemiological study [24]. Although the correlation between VMS and obesity has been repeatedly reported, it should be further studied in relation to central obesity, body mass index (BMI)-defined obesity, and visceral obesity, which may be diversely related to metabolic diseases [25]. There is evidence of association between VMS and high blood pressure [26] and insulin resistance [10]; however, these findings should be confirmed with large studies.

Several mechanisms linking VMS and metabolic syndrome have been suggested. A decreased endogenous serum estrogen level in the menopausal period seems to be linked with several metabolic impairment profiles; however, it cannot explain the pathophysiology of VMS [27]. Separately, chronic sympathetic nervous system activation is associated with a narrowed thermoneutral zone in the brain that may cause VMS and result in metabolic disturbances including altered vascular function, changes in blood pressure and lipid level, and insulin resistance [11]. Obesity is a representative shared determinant factor of VMS and metabolic syndrome, although the association between them remained after adjustment for obesity or BMI [14]. However, evidence supporting these hypotheses is insufficient, and further investigations are warranted.

\section{VMS AND DM}

According to a previous study that prospectively examined the association between VMS and incident diabetes among 150,007 postmenopausal Women's Health
Initiative participants from 1993 to 2014, VMS was associated with an $18 \%$ increase in diabetes risk (95\% confidence interval, 1.14-1.22), while VMS severity was similarly associated with the risk of diabetes [28]. Of note, these associations remained following adjustment for obesity and were more pronounced in women reporting night sweats together with hot flashes than for those reporting hot flashes only [28]. An association between VMS and insulin resistance, a precursor of diabetes, was also reported by several epidemiologic studies $[10,29]$. In the SWAN study cohort of 3,075 women with eight years of follow-up, hot flashes were correlated with a higher homeostatic model assessment of insulin resistance score, a measure of insulin resistance, independent of estradiol level [29]. Obesity, sleep disturbance, and sex hormone level changes were suggested as linking factors, but the supporting evidence available to date is not sufficient. Mainly due to the small number of previous studies on the association of VMS with DM or insulin resistance, this issue requires elucidation in further large-scale studies.

\section{VMS AND CARDIOVASCULAR RISKS}

Nearly half of all deaths in women over 50 years of age are associated with CVDs [30]. Screening of high-risk populations and implementation of effective primary prevention measures are the most important healthcare priorities. The association between menopause and increased risk of CVDs in women has been welldescribed [31], and that between menopausal VMS and CVDs has also been repeatedly reported in more recent studies, although there is no casual evidence. A prospective cohort study of 11,725 women aged 45 to 50 years demonstrated that women with frequent VMS were at increased risk of developing coronary heart disease over 14 years after adjustment for age, menopause status, lifestyle, and other chronic diseases [32]. The SWAN study, one of the largest health studies among menopausal women, showed that women with VMS had indices of greater subclinical CVDs, including poorer endothelial function and poorer flow-mediated dilation, as well as greater aortic calcification and carotid artery intima media thickness (IMT) relative to women without VMS [33]. A recent large-scale metaanalysis assessing association between VMS and various cardiovascular risk markers also showed that VMS are associated with increased risk of CVDs, although this correlation was attenuated after adjustment for car- 
diovascular risk factors [34]. Sympathetic activation is the most frequently suggested mechanism linking VMS with CVDs. Chronic activation of the sympathetic nervous system seems to be associated with altered vascular function, changes in blood pressure and lipids, and development of insulin resistance [11].

Recent research, on the other hand, suggests that, apart from traditional CVD risk factors, relations between VMS and CVD risk are sensitive to timing and duration of VMS. In one study, the early-onset VMS group showed greater IMT results, which is a measure of subclinical CVDs [35]. In addition, women with a longer duration of VMS also experience more severe aortic calcification later in life [36]. The findings linking VMS and subclinical CVDs indicate that VMS occurring in early menopausal years and having a longer duration are associated with higher CVDs risk. Still, critical questions like the association of the mechanism of VMS as a marker of CVDs risk remain. Furthermore, relations between VMS and CVDs risk are not yet clear, so whether VMS are markers, cofactors, or part of a causal pathway of CVDs should be clarified. Future studies are warranted to reveal the exact mechanism of this correlation. For treating VMS, hormone therapy, e.g., supplementing estrogen, is considered the most effective method, but patients in this context could develop CVDs adversely, so the VMS and CVDs risk correlation should be judged carefully [37].

\section{VMS AND NAFLD}

NAFLD is a spectrum of liver disorders defined by the presence of excessive lipid accumulation, referred to as steatosis, in more than $5 \%$ of hepatocytes, with little or no alcohol consumption [38]. Menopause and low estrogen level have been reported to be risk factors of NAFLD [39]. Considering that NAFLD is a hepatic manifestation of metabolic syndrome and insulin resistance [40], and that VMS are correlated with those diseases, an association between VMS and NAFLD can be expected. However, few studies have examined the association between VMS and NAFLD. In a crosssectional study of 1,793 menopausal Korean women, moderate to severe VMS was significantly associated with risk of NAFLD (odds ratio, 1.50; 95\% confidence interval, 1.10-2.03) following adjustment for confounding factors including central obesity and insulin resistance [9]. Regarding the association between VMS and metabolic syndrome, obesity, sympathetic overactivity, and hypoestrogenism have been suggested as potential underlying mechanisms linking NAFLD and VMS [41]; however, evidence in this regard is lacking. Further longitudinal studies are warranted to confirm the association between VMS and NAFLD among menopausal women.

\section{VMS AND OSTEOPOROSIS}

Osteoporosis is a well-known chronic disease that is aggravated in menopausal women due to decreased serum estrogen level [42]. Previous large epidemiologic studies have consistently reported that middle-aged women with VMS had lower bone mineral density at the lumbar spine and/or femoral neck bone than did women without VMS [8,43-45]. However, other studies have presented conflicting results regarding this association [46]. Nevertheless, it is noteworthy that estrogenbased HRT for menopausal women with VMS can improve bone density or fracture risk [47]. Further clinical studies are needed to demonstrate that HRT may be a first-line therapy for prevention and/or treatment of osteoporosis in certain menopausal women with VMS.

\section{CONCLUSION}

Several previous studies have indicated that menopausal VMS are associated with increased risk of chronic diseases such as metabolic syndrome, type $2 \mathrm{DM}$, CVDs, NAFLD, and osteoporosis in perimenopausal and postmenopausal women (Fig. 2). Menopausal VMS are expected to be useful biomarkers or predictors of cardiovascular risk and these chronic diseases rather than just temporary symptoms in menopausal women. However, the casual relationship of VMS with these diseases and the exact underlying mechanism(s) have yet to be revealed. Further, we should ascribe importance to exploring these associations because of the effect of menopausal HRT on these chronic diseases as well as on VMS. Based on accumulating evidence, HRT for treatment of VMS might be a standard treatment for selected patients with VMS and an increased risk of these chronic diseases. On the contrary, HRT may have deteriorative effects in some patients with these diseases, such as heightened cardiovascular risk in specific populations; thus, further confirmative studies and prudent clinical application should be performed. Future research should aim to reveal the casual relationship of VMS with several chronic diseases, especially with 


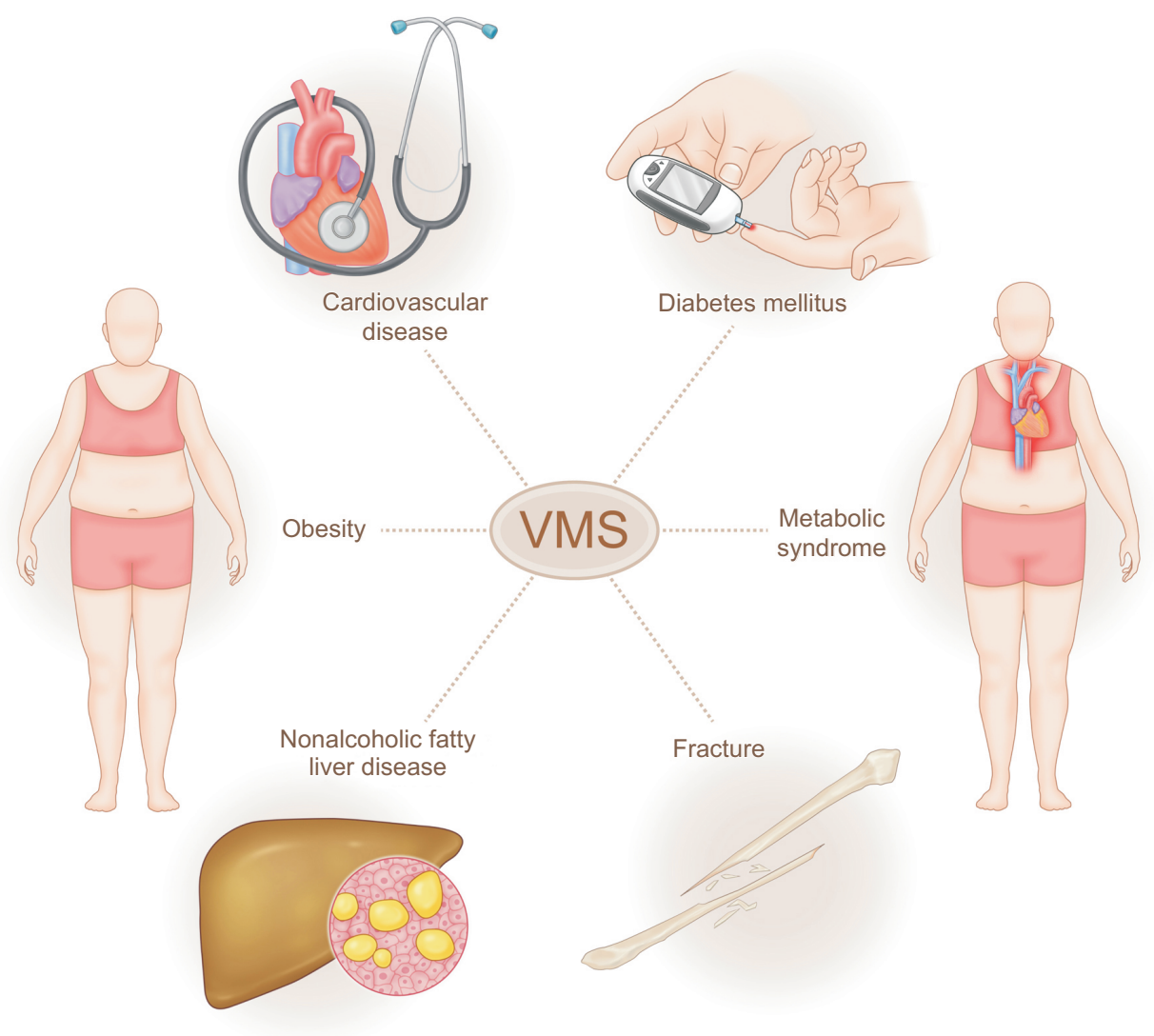

Fig. 2. Vasomotor symptoms (VMS) and chronic diseases in menopausal women. VMS are associated with increased risk of metabolic syndrome, insulin resistance, nonalcoholic fatty liver disease, osteoporosis, and cardiovascular diseases in menopausal women. reliable objective measurement of VMS. Analysis of big data also might assist in identifying the relationship of VMS with various factors in a real-world setting. More attention must be paid to the significance and management of VMS in middle-aged and older women.

\section{ACKNOWLEDGMENTS}

This work was supported by the National Research Foundation of Korea grant funded by the Korea government (MSIT) (No. 2020R1C1C1013579).

\section{CONFLICT OF INTEREST}

No potential conflict of interest relevant to this article was reported.

\section{REFERENCES}

1. Khang YH, Bahk J, Lim D, Kang HY, Lim HK, Kim YY, et al. Trends in inequality in life expectancy at birth between 2004 and 2017 and projections for 2030 in Korea: multiyear cross-sectional differences by income from national health insurance data. BMJ
Open 2019; 9: e030683.

2. Boulet MJ, Oddens BJ, Lehert P, Vemer HM, Visser A. Climacteric and menopause in seven south-east Asian countries. Maturitas 2008; 61: 34-53.

3. Avis NE, Crawford SL, Greendale G, Bromberger JT, Everson-Rose SA, Gold EB, et al. Duration of menopausal vasomotor symptoms over the menopause transition. JAMA Intern Med 2015; 175: 531-9.

4. Stuenkel CA, Davis SR, Gompel A, Lumsden MA, Murad MH, Pinkerton JV, et al. Treatment of symptoms of the menopause: an endocrine society clinical practice guideline. J Clin Endocrinol Metab 2015; 100: 3975-4011.

5. The NAMS 2017 Hormone Therapy Position Statement Advisory Panel. The 2017 hormone therapy position statement of The North American Menopause Society. Menopause 2017; 24: 72853.

6. Prague JK, Roberts RE, Comninos AN, Clarke S, Jayasena CN, Nash Z, et al. Neurokinin 3 receptor antagonism as a novel treatment for menopausal hot flushes: a phase 2, randomised, doubleblind, placebo-controlled trial. Lancet 2017; 389: 1809-20.

7. Lee SW, Jo HH, Kim MR, Kwon DJ, You YO, Kim JH. Association between menopausal symptoms and metabolic syndrome in postmenopausal women. Arch Gynecol Obstet 2012; 285: 541-8.

8. Ryu KJ, Park HT, Kim YJ, Yi KW, Shin JH, Hur JY, et al. Vaso- 
motor symptoms and osteoporosis in Korean postmenopausal women. Maturitas 2016; 87: 27-32.

9. Ryu KJ, Park H, Kim YJ, Yi KW, Shin JH, Hur JY, et al. Moderate to severe vasomotor symptoms are risk factors for non-alcoholic fatty liver disease in postmenopausal women. Maturitas 2018; 117: 22-8.

10. Kwon DH, Lee JH, Ryu KJ, Park HT, Kim T. Vasomotor symptoms and the homeostatic model assessment of insulin-resistance in Korean postmenopausal women. Obstet Gynecol Sci 2016; 59: 459.

11. Tuomikoski P, Savolainen-Peltonen H. Vasomotor symptoms and metabolic syndrome. Maturitas 2017; 97: 61-5.

12. Campagnoli C, Morra G, Belforte P, Belforte L, Prelato Tousijn L. Climacteric symptoms according to body weight in women of different socio-economic groups. Maturitas 1981; 3: 279-87.

13. Gast GC, Grobbee DE, Pop VJ, Keyzer JJ, Wijnands-van Gent CJ, Samsioe GN, et al. Menopausal complaints are associated with cardiovascular risk factors. Hypertension 2008; 51: 1492-8.

14. Ryu KJ, Park HT, Kwon DH, Yang KS, Kim YJ, Yi KW, et al. Vasomotor symptoms and metabolic syndrome in Korean postmenopausal women. Menopause 2015; 22: 1239-45.

15. Thurston RC, Chang Y, Mancuso P, Matthews KA. Adipokines, adiposity, and vasomotor symptoms during the menopause transition: findings from the Study of Women's Health across the Nation. Fertil Steril 2013; 100: 793-800.

16. Anderson GS, Martin AD. Calculated thermal conductivities and heat flux in man. Undersea Hyperb Med 1994; 21: 431-41.

17. Gold EB, Crawford SL, Shelton JF, Tepper PG, Crandall CJ, Greendale GA, et al. Longitudinal analysis of changes in weight and waist circumference in relation to incident vasomotor symptoms: the Study of Women's Health Across the Nation (SWAN). Menopause 2017; 24: 9-26.

18. Saccomani S, Lui-Filho JF, Juliato CR, Gabiatti JR, Pedro AO, Costa-Paiva L. Does obesity increase the risk of hot flashes among midlife women?: a population-based study. Menopause 2017; 24: 1065-70.

19. Luheshi GN, Gardner JD, Rushforth DA, Loudon AS, Rothwell NJ. Leptin actions on food intake and body temperature are mediated by IL-1. Proc Natl Acad Sci U S A 1999; 96: 7047-52.

20. Expert Panel on Detection, Evaluation, and Treatment of High Blood Cholesterol in Adults. Executive summary of the third report of The National Cholesterol Education Program (NCEP) expert panel on detection, evaluation, and treatment of high blood cholesterol in adults (adult treatment panel III). JAMA 2001; 285: 2486-97.

21. Lim S, Shin H, Song JH, Kwak SH, Kang SM, Won Yoon J, et al. Increasing prevalence of metabolic syndrome in Korea: the Korean National Health and Nutrition Examination Survey for 19982007. Diabetes Care 2011; 34: 1323-8.
22. Jouyandeh Z, Nayebzadeh F, Qorbani M, Asadi M. Metabolic syndrome and menopause. J Diabetes Metab Disord 2013; 12: 1.

23. Thurston RC, El Khoudary SR, Sutton-Tyrrell K, Crandall CJ, Gold EB, Sternfeld B, et al. Vasomotor symptoms and lipid profiles in women transitioning through menopause. Obstet Gynecol 2012; 119: 753-61.

24. Franco OH, Muka T, Colpani V, Kunutsor S, Chowdhury S, Chowdhury R, et al. Vasomotor symptoms in women and cardiovascular risk markers: Systematic review and meta-analysis. Maturitas 2015; 81: 353-61.

25. Sahakyan KR, Somers VK, Rodriguez-Escudero JP, Hodge DO, Carter RE, Sochor O, et al. Normal-weight central obesity: implications for total and cardiovascular mortality. Ann Intern Med 2015; 163: 827-35.

26. Vongpatanasin W. Autonomic regulation of blood pressure in menopause. Semin Reprod Med 2009; 27: 338-45.

27. Freedman RR. Pathophysiology and treatment of menopausal hot flashes. Semin Reprod Med 2005; 23: 117-25.

28. Gray KE, Katon JG, LeBlanc ES, Woods NF, Bastian LA, Reiber GE, et al. Vasomotor symptom characteristics: are they risk factors for incident diabetes? Menopause 2018; 25: 520-30.

29. Thurston RC, El Khoudary SR, Sutton-Tyrrell K, Crandall CJ, Sternfeld B, Joffe H, et al. Vasomotor symptoms and insulin resistance in the study of women's health across the nation. J Clin Endocrinol Metab 2012; 97: 3487-94.

30. Writing Group Members, Mozaffarian D, Benjamin EJ, Go AS, Arnett DK, Blaha MJ, et al. Heart disease and stroke statistics-2016 update: a report from the American Heart Association. Circulation 2016; 133: e38-360.

31. Muka T, Oliver-Williams C, Kunutsor S, Laven JS, Fauser BC, Chowdhury R, et al. Association of age at onset of menopause and time since onset of menopause with cardiovascular outcomes, intermediate vascular traits, and all-cause mortality: a systematic review and meta-analysis. JAMA Cardiol 2016; 1: 767-76.

32. Herber-Gast G, Brown WJ, Mishra GD. Hot flushes and night sweats are associated with coronary heart disease risk in midlife: a longitudinal study. BJOG 2015; 122: 1560-7.

33. Thurston RC, Sutton-Tyrrell K, Everson-Rose SA, Hess R, Powell LH, Matthews KA. Hot flashes and carotid intima media thickness among midlife women. Menopause 2011; 18: 352-8.

34. Muka T, Oliver-Williams C, Colpani V, Kunutsor S, Chowdhury S, Chowdhury R, et al. Association of vasomotor and other menopausal symptoms with risk of cardiovascular disease: a systematic review and meta-analysis. PLoS One 2016; 11: e0157417.

35. Thurston RC, El Khoudary SR, Tepper PG, Jackson EA, Joffe H, Chen HY, et al. Trajectories of vasomotor symptoms and carotid intima media thickness in the study of Women's Health across the Nation. Stroke 2016; 47: 12-7.

36. Thurston RC, Kuller LH, Edmundowicz D, Matthews KA. His- 
tory of hot flashes and aortic calcification among postmenopausal women. Menopause 2010; 17: 256-61.

37. Collins P, Rosano G, Casey C, Daly C, Gambacciani M, Hadji P, et al. Management of cardiovascular risk in the perimenopausal women: a consensus statement of European cardiologists and gynecologists. Climacteric 2007; 10: 508-26.

38. Sanyal AJ, Brunt EM, Kleiner DE, Kowdley KV, Chalasani N, Lavine JE, et al. Endpoints and clinical trial design for nonalcoholic steatohepatitis. Hepatology 2011; 54: 344-53.

39. Gutierrez-Grobe Y, Ponciano-Rodríguez G, Ramos MH, Uribe M, Méndez-Sánchez N. Prevalence of non alcoholic fatty liver disease in premenopausal, posmenopausal and polycystic ovary syndrome women. The role of estrogens. Ann Hepatol 2010; 9: 402-9.

40. Cobbina E, Akhlaghi F. Non-alcoholic fatty liver disease (NAFLD) - pathogenesis, classification, and effect on drug metabolizing enzymes and transporters. Drug Metab Rev 2017; 49: 197-211.

41. Sigala B, McKee C, Soeda J, Pazienza V, Morgan M, Lin CI, et al. Sympathetic nervous system catecholamines and neuropeptide $\mathrm{Y}$ neurotransmitters are upregulated in human NAFLD and modulate the fibrogenic function of hepatic stellate cells. PLoS One
2013; 8: e72928.

42. Cummings SR, Browner WS, Bauer D, Stone K, Ensrud K, Jamal $S$, et al. Endogenous hormones and the risk of hip and vertebral fractures among older women. Study of Osteoporotic Fractures Research Group. N Engl J Med 1998; 339: 733-8.

43. Min YK. Update on denosumab treatment in postmenopausal women with osteoporosis. Endocrinol Metab (Seoul) 2015; 30: 1926.

44. Gast GC, Grobbee DE, Pop VJ, Keyzer JJ, Wijnands-van Gent CJ, Samsioe GN, et al. Vasomotor symptoms are associated with a lower bone mineral density. Menopause 2009; 16: 231-8.

45. Ozkaya E, Cakir E, Kara F, Okuyan E, Cakir C, Ustün G, et al. Impact of hot flashes and night sweats on carotid intima-media thickness and bone mineral density among postmenopausal women. Int J Gynaecol Obstet 2011; 113: 235-8.

46. Huang A, Grady D, Blackwell T, Bauer D. Hot flushes, bone mineral density, and fractures in older postmenopausal women. Obstet Gynecol 2007; 109: 841-7.

47. Levin VA, Jiang X, Kagan R. Estrogen therapy for osteoporosis in the modern era. Osteoporos Int 2018; 29: 1049-55. 